\title{
OsWRKY13 Mediates Rice Disease Resistance by Regulating Defense-Related Genes in Salicylate- and Jasmonate-Dependent Signaling
}

\author{
Deyun Qiu, Jun Xiao, Xinhua Ding, Min Xiong, Meng Cai, Yinglong Cao, Xianghua Li, Caiguo Xu, and \\ Shiping Wang
}

National Key Laboratory of Crop Genetic Improvement, National Center for Plant Gene Research (Wuhan), Huazhong Agricultural University, Wuhan 430070, China

Submitted 2 October 2006. Accepted 3 December 2006.

\begin{abstract}
Although 109 WRKY genes have been identified in the rice genome, the functions of most are unknown. Here, we show that OsWRKY13 plays a pivotal role in rice disease resistance. Overexpression of $O S W R K Y 13$ can enhance rice resistance to bacterial blight and fungal blast, two of the most devastating diseases of rice worldwide, at both the seedling and adult stages, and shows no influence on the fertility. This overexpression was accompanied by the activation of salicylic acid (SA) synthesis-related genes and SA-responsive genes and the suppression of jasmonic acid (JA) synthesisrelated genes and JA-responsive genes. OsWRKY13 bound to the promoters of its own and at least three other genes in SA- and JA-dependent signaling pathways. Its DNA-binding activity was influenced by pathogen infection. These results suggest that OsWRKY13, as an activator of the SA-dependent pathway and a suppressor of JA-dependent pathways, mediates rice resistance by directly or indirectly regulating the expression of a subset of genes acting both upstream and downstream of SA and JA. Furthermore, OsWRKY13 will provide a transgenic tool for engineering wider-spectrum and whole-growth-stage resistance rice in breeding programs.
\end{abstract}

Additional keyword: Oryza sativa.

Plants have evolved an efficient defense transduction network against pathogen attack. Although many components of this network are still unknown, the salicylic acid (SA)-dependent systemic acquired resistance (SAR) pathway and the jasmonic acid and ethylene (JA/ET)-induced resistance pathway are well-recognized (Glazebrook 2001; Hammond-Kosack and Parker 2003). SA, JA, and ET are each involved in the regulation of defense against different pathogens (Thomma et al. 1998). Resistance against biotrophic pathogens is usually regulated by the SA-dependent pathway, whereas resistance against necrotrophic pathogens and insects is usually controlled by the JA/ET-dependent pathway (Felton and Korth 2000). However, SA- and JA/ET-dependent defense signals interact with each other, either synergistically or antagonistically (Durrant and Dong 2004). Activation of SAR can suppress JA biosynthesis and JA-responsive gene expression, and SA deficiency can

Corresponding author: S. Wang; Telephone: +86-27-87283009; Fax: +8627-87287092; E-mail: swang@mail.hzau.edu.cn

*The $\boldsymbol{e}$-Xtra logo stands for "electronic extra" and indicates that three supplemental tables, two supplemental figures, and supplemental methods information are available online and that Figures 1 and 3 are in color online. result in accumulation of JA and enhanced expression of JAresponsive genes.

NPR1 is a regulatory protein in Arabidopsis thaliana that modulates pathogenesis-related $(P R)$ gene expression, resulting in activation of SAR through binding to members of the TGA class of basic leucine-zipper transcription factor family. NPR1 is an important regulator in the antagonistic cross-talk between the two induced resistance pathways (Pieterse and Van Loon 2004; Zhang et al. 1999). Nuclear localization of NPR1 is required for SA-mediated defense gene expression (Kinkema et al. 2000), whereas suppression of JA signaling does not require nuclear localization of NPR1 (Spoel et al. 2003), indicating that NPR1 is not a direct regulator of JAresponsive gene expression.

Transcription activation of SA- and JA-responsive genes is essential for the induced resistance conferred by the two pathways. In addition to TGAs, WRKY transcription factors are also involved in modulation of SA- or JA-responsive gene expression and disease resistance (Asai et al. 2002; Chen and Chen 2002; Deslandes et al. 2002; Kim et al. 2006; Noutoshi et al. 2005; Robatzek and Somssich 2002; Xu et al. 2006; Yang et al. 1999; Zheng et al. 2006) in Arabidopsis and tobacco. WRKY70 is a node of convergence for integrating SA- and JA-mediated signals during Arabidopsis response to pathogens (Li et al. 2004). The WRKY proteins are a class of regulators that control diverse developmental and physiological processes in addition to disease-resistance responses (Eulgem et al. 2000). The rice WRKY superfamily consists of about 109 members, although the functions of most are unknown $(\mathrm{Qu}$ and Zhu 2006). Thus far, there has been no direct study of the role of rice WRKY protein in pathogen-induced defense regulation, although a few pathogen-induced WRKY genes have been identified (Liu et al. 2005b; Wen et al. 2003).

Bacterial blight caused by Xanthomonas oryzae pv. oryzae and fungal blast caused by Magnaporthe grisea are two of the most devastating diseases of rice worldwide. Our previous study showed that the cDNA clone EI12I1, corresponding to OsWRKY13 of the rice WRKY numbering system of Xie and associates (2005), increased expression after incompatible pathogen infection of several rice lines carrying different resistance $(R)$ genes conferring resistance for $X$. oryzae pv. oryzae or M. grisea; no expression change of EI12I1 was observed in compatible interactions (Wen et al. 2003). In addition, OsWRKY13 (EI12I1) colocalizes with a resistance quantitative trait locus against blast disease in rice chromosome 1 (Wen et al. 2003). Thus, we propose two hypotheses regarding the function of $O s W R K Y 13$. First, it may be involved in rice resis- 
tance in a pathogen nonspecific way, but activation of $R$ genes is the key to initiate its function. Second, constitutive overexpression of $O s W R K Y 13$ may enhance the defense of rice against diseases, regardless of the existence of an $R$ gene.

To examine the hypotheses, we monitored OsWRKY13 expression. Here, we show that overexpression of $O s W R K Y 13$ in the genetic background without an $R$ gene enhanced rice resistance to both $X$. oryzae pv. oryzae and $M$. grisea. In addition to phenotypic changes, OsWRKY13-overexpressing plants also showed expression profiling variation of a subset of defense-related genes, which were responsive to either JA, SA, or both, or were known to function in JA- or SA-dependent pathways. OsWRKY13 showed different DNA-binding abilities before and after pathogen challenge. The expressional characteristics of the defense-related genes in the transgenic plants and the interaction of OsWRKY13-DNA suggest that this protein mediates resistance by simultaneous direct or indirect activation of the genes in the SA-dependent pathway and suppression of the genes in the JA-dependent pathway.

\section{RESULTS}

Os WRKY13 modulates rice resistance

to both bacterial blight and fungal blast.

Comparative analysis of the genomic and cDNA sequences of OsWRKY13 (GenBank accession number EF143611) from resistant cultivar Minghui 63 (Oryza sativa subsp. indica) showed that the gene was 1,505 bp in length and had a coding region interrupted by two introns (Supplemental Fig. 1A). The putative encoding product of OsWRKY13 consisted of 316 amino acids, which contained a WRKY motif, a zinc-finger motif, and a potential nuclear localization signal (Fig. 1A). According to the classification of the WRKY superfamily (Eulgem et al. 2000), OsWRKY13 belongs to group II.

$O s W R K Y 13$ was overexpressed in cultivar Mudanjiang $8(O$. sativa subsp. japonica). Minghui 63 was moderately resistant to $X$. oryzae pv. oryzae PXO61, although it carried two genes, $X a 25(t)$ and $X a 26$, for bacterial blight resistance. Mudanjiang 8 , without any known $R$ gene, was highly susceptible to $X$. oryzae pv. oryzae. At the booting stage, 13 of the 47 plants showed significantly enhanced resistance to PXO61, with the lesion area ranging from 24.4 to $48.6 \%$ versus 61.8 and $32.2 \%$ for the controls of Mudanjiang 8 and Minghui 63, respectively. The enhanced resistance was stably inherited (Fig. 1B). The reduced lesion area of transgenic plants after $X$. oryzae pv. oryzae inoculation was associated with increased accumulation of OsWRKY13 transcripts (Fig. 1C, Supplemental Table 1).

To verify that the resistance of the transgenic plants was due to overexpression of $O s W R K Y 13$, four $\mathrm{T}_{2}$ families (D11UM14, D11UM7-2, D11UM18-9, D11UM18-10) from three resistant $\mathrm{T}_{0}$ plants (D11UM1, D11UM7, D11UM18) were examined individually at the booting stage for resistance by inocula-
A

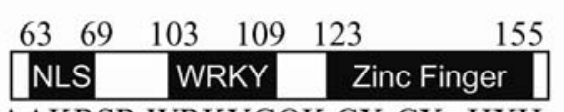
PAAKRSR WRKYGQK $\mathrm{CX}_{5} \mathrm{CX}_{23} \mathrm{HXH}$

B

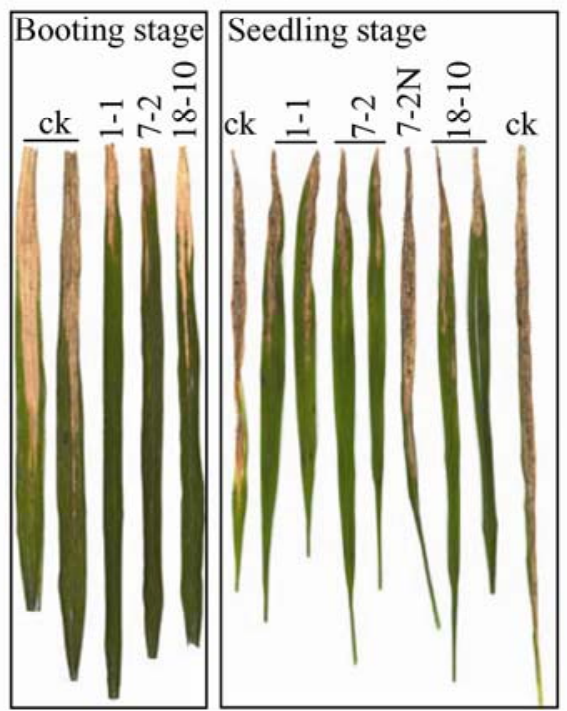

C

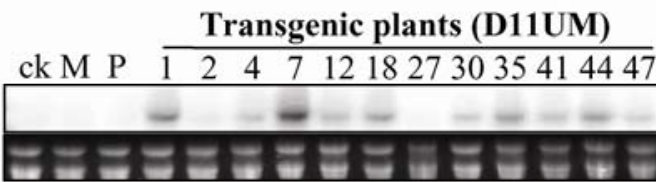

D

E
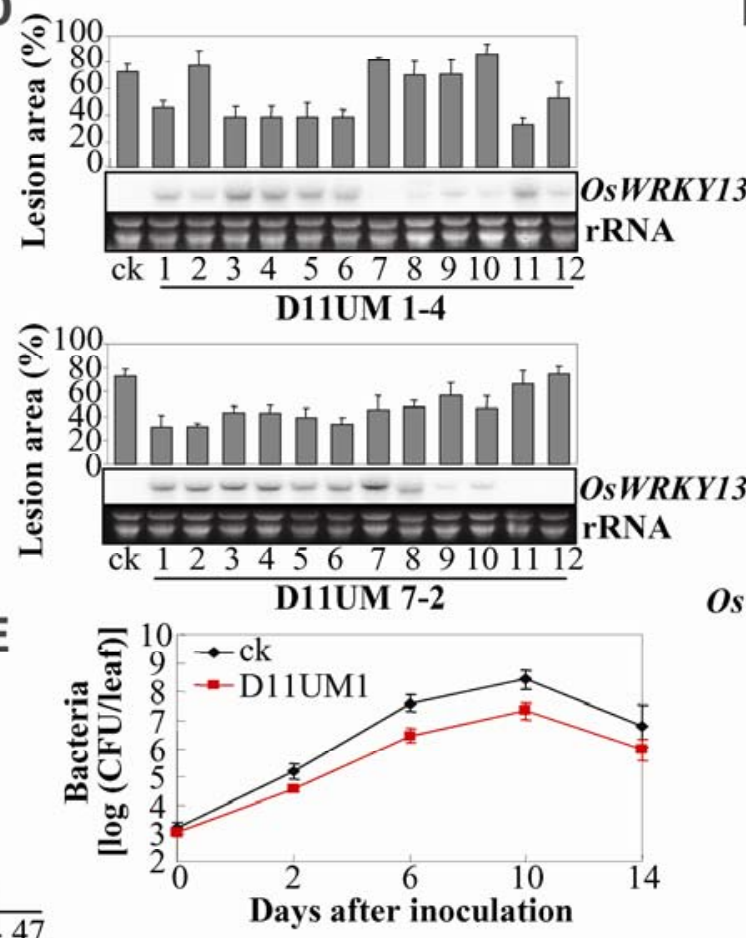

OsWRKY13

RRNA
$\mathbf{F}$

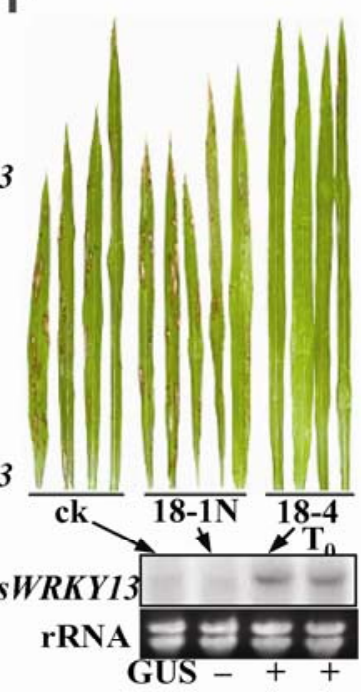

Fig. 1. Product and function of the $O s W R K Y 13$ gene. A, Schematic diagrams of conserved motifs of OsWRKY13. NLS = nuclear localization signal, WRKY = WRKY motif, and zinc finger = zinc-finger motif. Conserved sequences of these structures are presented, and numbers indicate the amino acid position of each structure in OsWRKY13. B, OsWRKY13-overexpressing plants show enhanced resistance to Xanthomonas oryzae pv. oryzae PXO61 at both the seedling and booting stages. The $\mathrm{T}_{2}$ transgenic plants $(1-1,7-2,18-10)$ are from $\mathrm{T}_{0}$ plants D11UM1, D11UM7, and D11UM18. 7-2N is a transgene-negative plant segregated in the D11UM7-2 family. C, Expression analysis of OsWRKY13 in noninoculated $\mathrm{T}_{0}$ transgenic plants, wild type, mock (water)-inoculated Minghui 63 (donor of $O s W R K Y 13, \mathrm{M}$ ), and PXO61-inoculated Minghui 63 (P) by RNA gel blot analysis. D, Enhanced resistance to PXO61 cosegregates with overexpression of $O s W R K Y 13$ in $\mathrm{T}_{2}$ families D11UM1-4 and D11UM7-2. E, Growth of PXO61 in leaves of T ${ }_{3}$ s $W R K Y 13$-overexpressing (D11UM1) and wild-type plants. Bacterial populations were determined from three leaves at each timepoint by counting CFUs. Similar results were obtained in two independent biological experiments. F, Resistance of $O s W R K Y 13$-overexpressing plants for Blast. The $\mathrm{T}_{1}$ negative (D11UM18-1N) and positive (D11UM18-4) plants were from $\mathrm{T}_{0}$ plant D11UM18. Similar results were obtained in two independent biological experiments. ck = Mudanjiang 8 (wild type). 
tion with PXO61 and measuring OsWRKY13 expression level. The enhanced resistance cosegregated with the increased level of $O s W R K Y 13$ transcripts in all $\mathrm{T}_{2}$ families (Fig. 1D, result from only two families shown). The growth rate of bacteria on resistant transgenic plants was 5.5- to 13.9-fold lower than that on susceptible control Mudanjiang 8 at 2 to 14 days after inoculation (Fig. 1E). The resistance of OsWRKY13-overexpressing plants to PXO61 at the four- to five-leaf stage was also enhanced significantly, as evaluated by lesion area (Fig. 1B). These results suggest that overexpression of OsWRKY13 can improve resistance against bacterial blight disease at both the seedling and adult stages.

Mudanjiang 8 was susceptible to $M$. grisea, which causes blast disease. After infection with $M$. grisea at the tillering stage in a natural infection field, the $\mathrm{T}_{1}$ plants from $O s W R K Y 13$-overexpressing $\mathrm{T}_{0}$ plant D11UM18 segregated into two groups, a highly susceptible group that had numerous lesions on the leaves and a partly resistant group that had few lesions on the leaves. The enhanced resistance of the plants cosegregated with both the overexpression of $O s W R K Y 13$ and the expression of $\beta$ glucuronidase, which represented the presence of the transgene (Fig. 1F). The $\mathrm{T}_{1}$ plants from $\mathrm{T}_{0}$ plant D11UM47 were also examined for blast resistance by inoculation with $M$. grisea isolate V86013 in a greenhouse at the three- to four-leaf stage. Again, the enhanced resistance of $\mathrm{T}_{1}$ plants cosegregated with overexpression of the $O s W R K Y 13$ gene (data not shown). These results suggest that $O s W R K Y 13$ was also involved in blast resistance at both the seedling and adult stages.

\section{OsWRKY13 regulates the expression of other defense-related genes.}

The expression patterns of two $P R$ genes and eight genes known to function in SA- or JA-dependent defense signaling pathways in an OsWRKY13-overexpressing (D11UM7-2) line were examined. Six of the ten genes showed differential expression upon pathogen challenge in susceptible wild-type plant Mudanjiang 8. PXO61 challenge significantly induced the expression of PRla (acidic PR protein, AJ278436), LOX (lipoxygenase, D14000), and AOS2 (allene oxide synthase 2, AY062258) and suppressed the expression of PR10 (ribonuclease, D38170), PAL (phenylalanine ammonia-lyase, X87946), and $C H S$ (chalcone synthase, X89859) in Mudanjiang 8 (Fig. 2). The expression of NH1 (Arabidopsis NPR1 homolog 1, AY923983) and Arabidopsis homologs EDS1 (enhanced disease susceptibility 1, AK100117), PAD4 (phytoalexin deficient 4, CX118864), and ICS1 (isochorismate synthase 1, AK120689) was not affected by pathogen inoculation in Mudanjiang 8.
OsWRKY13-overexpressing plants accumulated significantly more PRIa, PAD4, ICS1, and CHS transcripts and fewer AOS2 and PR10 transcripts than did the wild type (Fig. 2). Before pathogen infection, expression levels of $L O X$ and $N H I$ were significantly higher in OsWRKY13-overexpressing plants than in wild type. After inoculation, overexpressing OsWRKY13 suppressed $L O X$ and showed no significant influence on $\mathrm{NH}$, as compared with wild type (Fig. 2). The expression of EDS1 and $P A L$ was not significantly affected by the level of OsWRKY13 transcript.

\section{OsWRKY13 protein is targeted}

to the nucleus and binds to gene promoters.

To determine the subcellular localization of OsWRKY13, genomic DNA, and cDNA of OsWRKY13 was fused in frame with the gene encoding green fluorescence protein (GFP). The GFP-OsWRKY13 fusion gene driven by maize ubiquitin ( $\mathrm{Ubi}$ ) promoter was transiently expressed in onion epidermal cells. The recombinant fusion protein was localized in the nuclei (Fig. 3A to C), whereas cells bombarded with Ubi:GFP construct (control) yielded fluorescence in both cytoplasm and nuclei (Fig. 3D and E).

To determine if OsWRKY13 had DNA-binding activity, a series of yeast one-hybrid assays were performed using DNA fragments harboring $\mathrm{W}$ boxes (TTGACC/T), W-like boxes, or both (TTGACA, TGACC/T, or TTGAC) from the promoter regions of PRla, AOS2, LOX, ICS1, NH1, and PAD4. Detection of protein-DNA binding activity by growth performance on SD-Leu-Trp-His agar medium showed that OsWRKY13 possessed specific DNA-binding ability, binding strongly to the promoters of PRIa, AOS2, and LOX and not to the promoters of $I C S 1, N H 1$, and PAD4 (Fig. 3F and G).

The promoter of $O s W R K Y 13$ contains a TTGAC W-like box. A gel mobility shift assay showed that nuclear proteins from OsWRKY13-overexpressing plants had stronger binding signal to the probe, harboring the W-like box, than that from wild type (Fig. $3 \mathrm{H}$ ). Pathogen inoculation increased the DNA-binding activity of the nuclear proteins from wild-type plants and reduced the binding activity of the nuclear proteins from OsWRKY13-overexpressing plants (Fig. $3 \mathrm{H}$ ). The glutathione- $S$ transferase (GST)-OsWRKY13 fusion proteins but not GST also bound strongly to the DNA probe (Fig. 3I). The DNAbinding intensity of either OsWRKY13 or nuclear proteins was reduced by the competition of unlabeled probe, indicating the specificity of the binding. The W-like box is partly overlapped with the binding site (TGACG) for TGA transcription factors in the DNA probe. To determine the binding specificity, the DNA probe was site-mutated (Fig. 3J). Both OsWRKY13
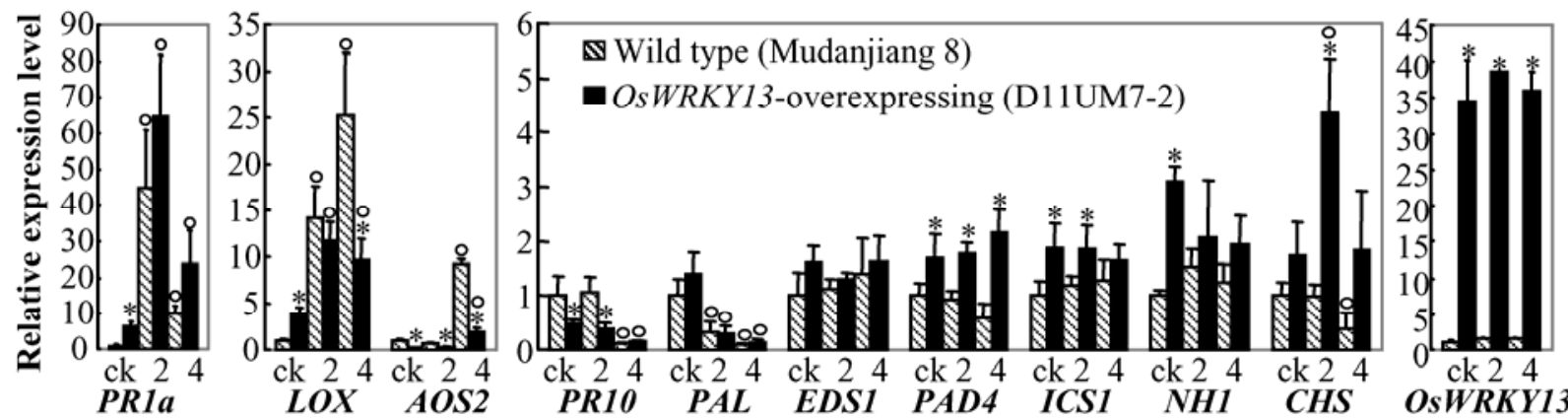

Fig. 2. Expression analysis of defense-related genes in $O s W R K Y 13$-overexpressing plants by quantitative reverse transcription-polymerase chain reaction. Samples were obtained before PXO61 inoculation (ck) and at 2 and 4 days postinoculation. The expression level of each gene in transgenic plants was calculated relative to that in noninoculated wild-type plants. Circles indicate a significant difference $(P<0.05)$ between noninoculated and inoculated plants and asterisks indicate a significant difference $(P<0.05)$ between the transgenic plant and corresponding wild type within the same treatment. The RNA sample for the transgenic plant was a mixture isolated from eight leaves of four WRKY13-overexpressing or wild-type plants. Similar results were obtained in two independent biological experiments. 
and nuclear proteins showed similar binding intensity to the mutated probe 1 , harboring a typical $\mathrm{W}$ box (TTGACC) and without TGA-binding site, as to the wild-type probe, but greatly reduced binding to the mutated probe 2 , without $\mathrm{W}$ or W-like box and TGA-binding site (Fig. 3J). These results suggest that both OsWRKY13 and the nuclear proteins bind specifically to the W-like box.
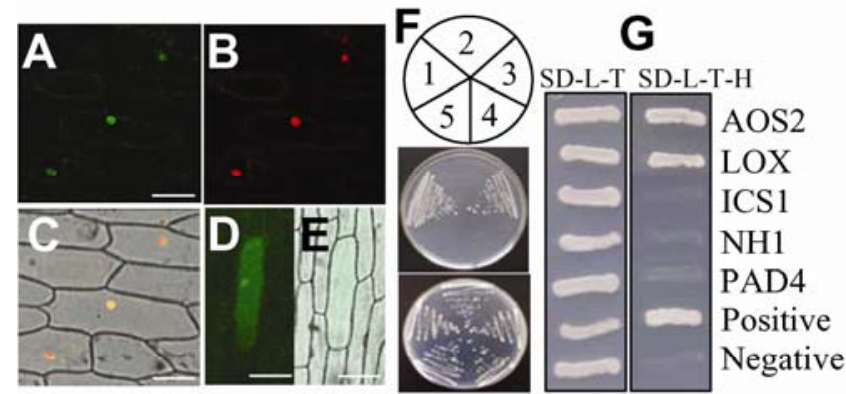
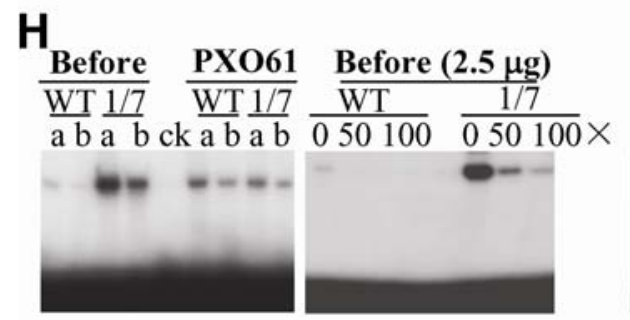

$1 / 7$ nuclear GSTJ Mutation site (lower case) protein OsWRKY13 $\mathrm{W} \mathrm{ml} \mathrm{m} 2 \mathrm{~W} \mathrm{~m} 1 \mathrm{~m} 2$

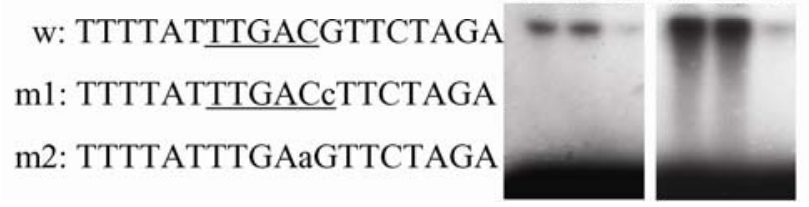

Fig. 3. Subcellular localization and DNA-binding activity of OsWRKY13. Photographs (A through E) of onion epidermal cells were taken under a confocal microscope. Scale bars $=100 \mu \mathrm{m}$. A, Green fluorescent protein (GFP)-OsWRKY13 expression in nuclei after incubation for $24 \mathrm{~h}$. B, Propidium iodide-stained nuclei. C, Transmission photograph overlaying A and B. D, GFP (control) expression in cytoplasm and nucleus. E, Transmission photograph overlaying D. F, Yeast one-hybrid assay using OsWRKY13 as target protein and target DNA (PR1a) with three W boxes from the promoter of PRIa and control DNA (W17) without a W box from the promoter of $O s W R K Y 13$ as baits. Top, schematic distribution of yeast cells carrying different plasmids; 1 , positive control (p53HIS2 plus pGAD-Rec2-53); 2, target DNA only; 3, OsWRKY13 plus target DNA; 4 OsWRKY13 plus control DNA (W17); 5, control DNA (W17) only. Middle, positive interaction between OsWRKY13 and target DNA PR1a was verified in SD-Leu-Trp-His (SD-L-T-H) agar medium with $30 \mathrm{mM} 3-$ amino-1, 2, 4-triazole. Bottom, yeast cells carrying different plasmids were grown for 2 days at $30^{\circ} \mathrm{C}$ in SD-Leu-Trp (SD-L-T) agar medium. G, Yeast one-hybrid assay using OsWRKY13 as target protein and target DNA fragments from the promoters of AOS2, LOX, ICS1, NH1, and PAD4 as baits. Positive control (p53HIS2 plus pGAD-Rec2-53); negative control (OsWRKY13 only). H, Nuclear proteins bind to DNA harboring a W-like box by gel mobility shift assays. ck = without nuclear protein. Nuclear proteins were isolated from wild-type (Mudanjiang 8, WT) and OsWRKY13-overexpressing $\mathrm{T}_{3}$ (D11UM1 and D11UM7, 1/7) plants at two days after PXO61-inoculation (PXO61) or before pathogen inoculation (Before). The "a" and "b" indicate 5- and 2.5- $\mu \mathrm{g}$ nuclear proteins, respectively. The labeled probes with or without 50 - or 100 -fold $(x)$ unlabeled probes were used for the binding assay. I, OsWRKY13 binds to DNA harboring a W-like box by gel mobility shift assays. Aliquots $(0.5$ $\mu \mathrm{g}$ ) of glutathione- $S$-transferase (GST) or GST-OsWRKY13 were used for DNA binding. J, Mutation (m2) of W-like box (underlined) in the original DNA probe $(\mathrm{w})$ influences protein binding in gel mobility shift assay. All of the binding assays were repeated at least twice with similar results.
OsWRKY13 influences the accumulation of SA.

Quantification of free SA and its conjugate, SA $\beta$-glucoside, showed that OsWRKY13-overexpressing plants accumulated more free SA than did wild-type plants, whereas wild-type plants had more SA $\beta$-glucoside (Fig. 4A).

\section{Defense signal molecules influence the expression of OsWRKY13.}

The universal defense signal molecules SA, its analogs thiadiazole-7-carbothioc acid $S$-methyl ester (BTH), and 2,6dichloroisonicotinic acid (INA), and JA and ethephon (an ethylene generator) all influenced the expression of OsWRKY13 (Fig. 4B). BTH induced OsWRKY13 expression at $5 \mathrm{~min}$ and its effect on OsWRKY13 expression began to disappear at 60 min after treatment, whereas SA and INA continually activated OsWRKY13 expression. The induced expression of OsWRKY13 began at $5 \mathrm{~min}$, and this effect began to disappear at $30 \mathrm{~min}$ after JA treatment. The activation of OsWRKY13 stimulated by ethephon showed a similar pattern as that of BTH.

\section{DISCUSSION}

Several WRKY proteins are involved in disease resistance of dicot plants (Chen and Chen 2002; Journot-Catalino et al. 2006; Kim et al. 2006; Li et al. 2004; Robatzek and Somssich 2002; Yang et al. 1999; Zheng et al. 2006) and many WRKY genes have been identified in the rice genome (Liu et al. 2005b; Qu and Zhu 2006; Xie et al. 2005). However, OsWRKY13 is the first WRKY protein characterized from rice that plays a pivotal role in disease resistance. Constitutive

\section{A}

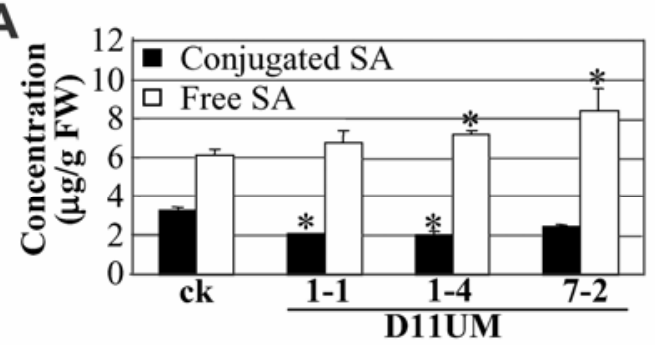

B

OsWRKY13

rRNA

$05153060901200515306090120 \mathrm{~min}$

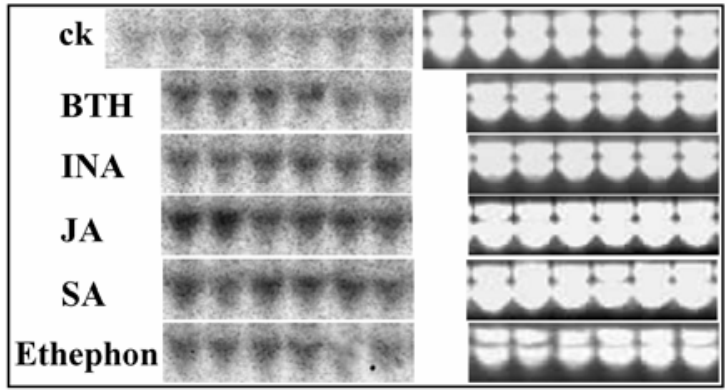

Fig. 4. Salicylic acid (SA) level and the effect of signal molecules. A, OsWRKY13 influences the accumulation of free SA and conjugated SA levels in the leaves of Mudanjiang 8 (ck) and OsWRKY13-overexpressing $\mathrm{T}_{2}$ plants, D11UM1-1, D11UM1-4, and D11UM7-2. Asterisks indicate a significant difference $(P<0.05)$ between transgenic and wild-type plants. Each sample was from four to eight plants. Similar results were obtained in two independent biological experiments. $\mathrm{FW}=$ fresh weight. B, Defense signal molecules influence OsWRKY13 expression in Minghui 63. The control (ck) plants were treated with water. Each sample contained ten leaf segments and was collected before treatment (0) or after treatment with thiadiazole-7-carbothioc acid $S$-methyl ester (BTH), 2,6-dichloroisonicotinic acid (INA), jasmonic acid (JA), salicylic acid (SA), or ethephon (an ethylene generator). Similar results were obtained in two independent biological experiments. 
expression of $O s W R K Y 13$ can enhance rice resistance to bacterial blight and fungal blast at both the seedling and adult stages and influences the expression of defense-related genes in SAand JA-dependent signaling. Thus, characterization of OsWRKY13 gives a crucial view to the regulation of pathogeninduced defense transduction pathways in rice.

\section{OsWRKY13 regulates a subset of defense-related genes in different signaling transduction pathways.}

The WRKY superfamily regulates gene expression by binding to the $\mathrm{W}$ or W-like box (Eulgem et al. 2000). The nuclear localization and DNA-binding ability of OsWRKY13 implies its role in the regulation of gene expression. The differential expression of eight of the ten defense-related genes in the SAor JA-dependent pathways in OsWRKY13-overexpressing plants further suggest the control of gene expression by OsWRKY13. Sequence analysis demonstrated that these eight defenserelated genes and $O s W R K Y 13$ were clustered with W or W-like boxes in their promoter regions (Supplemental Fig. 2). Binding of OsWRKY13 to the promoter regions of at least four genes (OsWRKY13, LOX, AOS2, PRla) was confirmed. Thus, resistance conferred by OsWRKY13 may be activated by its regulation of some defense-related genes through direct binding to their cis-elements.

We compared the whole-genome expression profiles of OsWRKY13-overexpressing (two independent lines) and wildtype plants using Affymetrix rice genome arrays (D. Qiu and S. Wong, unpublished data). A total of 26 transcription factors exhibited differential expression in OsWRKY13-overexpressing plants, based on the analysis with an estimated false discovery rate of $<5 \%$ and a cutoff value of twofold change (Hu and Ma 2006; Tusher et al. 2001). This result suggests that the differential expression of some defense-related genes in OsWRKY13-mediated resistance may also be through the action of other transcription factors regulated by OsWRKY13.

OsWRKY13 and nuclear proteins from OsWRKY13-overexpressing plants bind specifically to the $\mathrm{W}$ or W-like box (Fig.

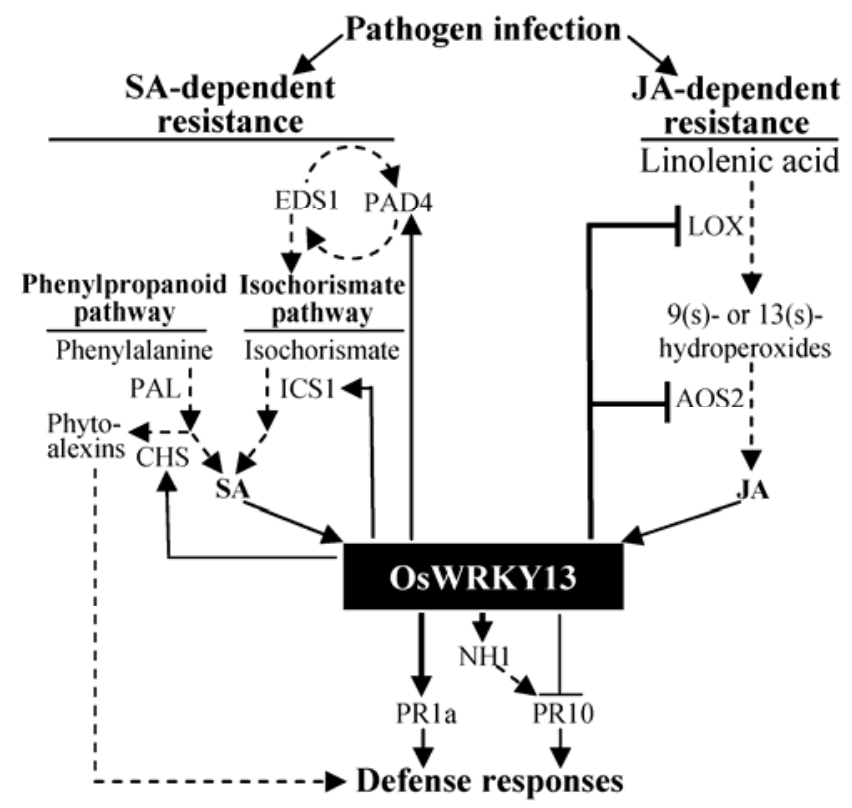

Fig. 5. Defense signaling model for the roles of OsWRKY13 in regulation of salicylic acid (SA)- and jasmonic acid (JA)-dependent pathways. Thick solid lines indicate direct action of OsWRKY13 has been confirmed by protein-DNA binding assays; thin solid lines, regulation may be executed by direct or indirect action; dotted lines, regulation has been confirmed in rice or other plant species; the line ending with arrow, activation; and the line ending with perpendicular short line indicates suppression.
3J). Nuclear proteins from OsWRKY13-overexpressing plants demonstrated stronger DNA-binding activity than that from wild type in the absence of pathogen challenge (Fig. $3 \mathrm{H})$. No WRKY genes (except OsWRKY13) or TGA genes in OsWRKY13-overexpressing plants showed enhanced expression based on rice whole-genome array analysis (D. Qiu and S. Wong, unpublished data). These results suggest that the DNAbinding activity of nuclear proteins may be mainly due to the action of OsWRKY13. Not all the eight genes under the control of OsWRKY13 showed similar expression patterns in OsWRKY13-overexpressing plants before and after pathogen challenge. For example, the expression level of $L O X$ was significantly higher before bacterial infection and lower after bacterial infection in $O s W R K Y 13$-overexpressing plants than that in wild type. Pathogen challenge influenced the DNA-binding activity of OsWRKY13. These results suggest that OsWRKY13 may have two functional conformations. The conformational change is associated with pathogen invasion that may modify the phosphorylation status of OsWRKY13. Previous studies have also reported that activation of certain WRKY transcription factors by phosphorylation or other modification is required for successful defense (Asai et al. 2002; Turck et al. 2004; Yang et al. 1999).

Some of the defense-related genes under direct or indirect control of OsWRKY13 were apparently also regulated by one or more other factors in OsWRKY13-mediated resistance. For example, although the expression of $L O X$ and AOS 2 was reduced in OsWRKY13-overexpressing plants as compared with that of wild type upon pathogen infection, the relative expression of these two genes increased significantly as compared with uninfected OsWRKY13-overexpressing plants (Fig. 2). Overexpression of $\mathrm{NHI}$ enhanced the expression of PRIO (Chern et al. 2005), but the expression pattern of $N H 1$ in OsWRKY13-overexpressing plant was not the same as that of $P R 10$. These results indicate that one or more other factors independent of OsWRKY13 may also regulate some of the defense-related genes. Furthermore, the differential expression of some of the defense-related genes in the transgenic plants could be influenced by the non-true in vivo binding of OsWRKY13 to the W or W-like box of their promoters due to the high nonphysiological concentration of OsWRKY13 in the cells. Thus, comparative analysis of the expression of these genes using an OsWRKY13-loss of function mutant will decipher the physiological regulatory function of OsWRKY13.

\section{OsWRKY13 is involved in the regulation} of both SA- and JA-dependent pathways.

NPR1 is a modulator for the antagonistic interaction of SAand JA-mediated signals in the disease resistance of Arabidopsis (Pieterse and Van Loon 2004), and it is a key regulator of SAR in Arabidopsis (Durrant and Dong 2004). NPR1 functions downstream of SA by regulating the expression of SAresponsive genes. The functional position of NPR1 in the JAdependent pathway is unclear, but it is not directly involved in the regulation of JA-responsive gene expression. The Arabidopsis WRKY protein WRKY70 was identified as a common component in the SA- and JA-dependent pathways ( $\mathrm{Li}$ et al. 2004), regulating the antagonistic interaction of the two defense transduction pathways. WRKY70 is downstream of NPR1 and activates SA-responsive genes; it is also downstream of JA and suppresses JA-responsive genes. Overexpression of NPR1 or rice NPR1 homolog NH1 can enhance rice resistance to $X$. oryzae pv. oryzae; in addition, NPR1 and NH1 interact with rice TGA proteins, suggesting that rice and Arabidopsis share a conserved signal transduction pathway controlling NPR1-mediated resistance (Chern et al. 2001, 
2005). However, it is unclear whether NPR1 or NH1 also regulate the interaction of SA- and JA-mediated signals in rice disease resistance. The present data indicate that OsWRKY13, functioning upstream of NH1, may be involved in the antagonistic interaction between SA- and JA-dependent pathways by regulating the expression of a subset of genes involved in synthesis of SA, JA, and PR proteins through multiple positive and negative feedback loops. A proposed functional model of OsWRKY13 in rice defense responses is shown in Figure 5.

Unlike Arabidopsis WRKY70, whose expression is activated by SA and suppressed by JA (Li et al. 2004), expression of $O s$ WRKY13 was induced by both SA and JA. OsWRKY13 expression affected the transcript levels of $P R$ genes and $N H 1$. Thus, one action site of OsWRKY13 should be downstream of SA and JA and upstream of NH1 and PR proteins in the defense signaling network (Fig. 5). Both SA and JA can induce the expression of PRI $a$ and PR10 in rice (Agrawal et al. 2000, 2001). However, the expression patterns of these $P R$ genes in transgenic plants (Fig. 2) clearly indicated that OsWRKY13 activates PRIa and suppresses PR10. PRI expression is the marker of SAR (Durrant and Dong 2004). PRla showed the similar expression pattern as the genes, such as $P A D 4$ and ICS1, putatively functioning in SA-dependent pathway. Therefore, PRla may function in the SA-dependent pathway, whereas PR10 most likely functions in the JA-dependent pathway because its expression pattern is similar to those of $L O X$ and AOS 2 in JA signaling in OsWRKY13-mediated resistance (Figs. 2 and 5).

SA can be synthesized via the isochorismate pathway and the phenylpropanoid pathway (Fig. 5) (Lee et al. 1995; Mauch et al. 2001). The isochorismate pathway appears to be the main source of SA synthesis during SAR, and the phenylpropanoid pathway appears to be responsible for the rapid production of SA associated with local cell death in dicot plants (Durrant and Dong 2004; Wildermuth et al. 2001). ICS1 is a key enzyme for SA synthesis in the isochorismate pathway. Two other proteins required for some $R$ gene-mediated resistance, PAD4 and EDS1, function in a positive feedback loop to regulate SA synthesis upstream of ICS1 in dicot plants (Falk et al. 1999; Feys et al. 2001; Jirage et al. 1999). Although no study has examined the involvement of EDS1/PAD4 in the isochorismate pathway in rice, evidence supports the existence of an SA synthesis pathway during $\mathrm{SAR}$ in rice similar to that in dicot plants. First, enhanced expression of PAD4 and ICS1 was accompanied by an increased level of free SA in OsWRKY13-overexpressing plants. Second, incompatible interaction induced $P A D 4$ expression, implying its role in $R$ gene-mediated resistance (D. Qiu and S. Wong, unpublished data). CHS functions on a branch of the phenylpropanoid pathway and catalyzes synthesis of defensive secondary metabolites, such as phytoalexins, using the intermediate of SA synthesis as a matrix (Gozzo 2003). Overexpressing OsWRKY13 also enhanced the expression of $C H S$. These results suggest that other action sites of OsWRKY13 in the SA-dependent pathway are upstream of PAD4 and CHS and activate the synthesis of SA and phytoalexins, respectively, through a feedback control loop.

The JA synthesis pathway begins with $\alpha$-linolenic acid, and LOX and AOS are two important enzymes in this pathway (Fig. 5) (Zhao et al. 2005). AOS2 is important in accumulation of JA in rice (Mei et al. 2006). In OsWRKY13-overexpressing plants, the expression of $L O X$ and AOS2 was suppressed accompanying the activation of genes functioning in the SA-dependent pathway upon pathogen infection, indicating that OsWRKY13 may suppress the JA-dependent pathway by negative feedback regulation of JA synthesis.

\section{Conclusion.}

Some components involved in the cross-talk between SAand JA-dependent defense pathways against pathogens and insects have been revealed in dicot plants, but how this interaction is regulated in rice, which has a high basal SA level, is unknown. The present results show that OsWRKY13 can directly or indirectly regulate the expression of genes functioning upstream and downstream of SA and JA, providing a starting point for studying the interaction between SA- and JAdependent pathways during rice disease resistance. Overexpression of OsWRKY13 can enhance rice resistance to both bacterial and fungal pathogens without causing deleterious fertile effects. Thus, this protein also provides a transgenic tool for engineering wider-spectrum and whole-growth-stage resistance rice in breeding programs.

\section{MATERIALS AND METHODS}

\section{Gene isolation and structural analysis.}

EI12I1 is the cDNA fragment of the OsWRKY13 gene. A genomic bacterial artificial chromosome (BAC) library constructed with Minghui 63 tissues was screened using EI12I1 as probe, and a positive BAC clone, 7G7, was identified. A fragment about $3.8 \mathrm{~kb}$ in length and harboring OsWRKY13 was obtained from $7 \mathrm{G} 7$ by digestion with restriction enzyme HindIII and was subcloned into vector pUC19, and the subclone (7G7H14) was sequenced. The structure of OsWRKY13 was determined by sequencing the transcript of the gene. The $3^{\prime}$ untranslated region was analyzed by $3^{\prime}$ rapid amplification of cDNA end (RACE) assays using the 3' Full RACE core set (TaKaRa Biotechnology Co., Ltd., Dalian, China), according to the manufacture's protocols, and OsWRKY13-specific primer WRKY4 (5'-GGATGGATCGACCAGTACCA-3'). The $5^{\prime}$ untranslated region was determined by reverse transcriptionploymerase chain reaction (RT-PCR), using primers WRKY14F (5'-CTGCGCTTAGTTTGTGGGTGGG-3') and WRKY6R (5'GGAGGTGATCATCAGGAG-3'), designed according to the full-length cDNA of the OsWRKY13 allele in rice cultivar Nipponbare (GenBank accession number AK067329).

\section{Plant transformation.}

The overexpression construct of OsWRKY13 was made by inserting a 3-kb DNA fragment digested with BamHI from clone 7G7H14 into vector pU1301, which was modified by insertion of a maize ubiquitin promoter and a napoline synthase polyadenylation signal (NOS) terminator into the multiple cloning sites of vector pCAMBIA1301. Agrobacterium-mediated transformation was performed according to the protocol of Lin and Zhang (2005).

\section{Pathogen inoculation.}

To evaluate bacterial blight disease, plants were inoculated with X. oryzae pv. oryzae PXO61 at the seedling or booting stage by the leaf-clipping method (Kauffman et al. 1973). Disease was scored by measuring the percent lesion area (lesion length/leaf length) at 2 to 3 weeks after inoculation. For blast disease evaluation, seedlings at the three- to four-leaf stage were inoculated with $M$. grisea isolate V86013 by the spraying method (Chen et al. 2001) or plants were infected in a natural infection nursery. Mock-inoculated (control) plants were treated under the same conditions, except that pathogen suspension was replaced with water.

\section{RNA gel blot and quantitative RT-PCR analyses.}

Aliquots $(20 \mu \mathrm{g})$ of total RNA were used for RNA gel blot analysis (Zhou et al. 2002). The cDNA fragment (EI12I1) of OsWRKY13 was used as a hybridization probe. RT-PCR was conducted as described by Wen and associates (2003). Quantitative PCR was performed, using the ABI 7500 real-time PCR system (Applied Biosystems, Foster City, CA, U.S.A.) accord- 
ing to the manufacturer's instruction. The PCR primers for all the genes are listed in Supplemental Table 2. The expression level of actin was used to standardize the RNA sample for each RT-PCR. The quantitative PCR reaction was in a $25-\mu$ l volume containing $1 \mu \mathrm{l}$ of diluted reverse transcription product, $12.5 \mu \mathrm{l}$ of $2 \times$ SYBR green PCR master mix (Applied Biosystems), and $0.32 \mu \mathrm{M}$ of each primer.

\section{Subcellular localization of OsWRKY13.}

OsWRKY13 was fused with GFP gene. The coding region was obtained by PCR amplification of the genomic DNA and the cDNA of OsWRKY13. The PCR products were cloned into vector pU1300. Transient expression of the fusion gene in white onion (Allium cepa) epidermal cells was performed, essentially as described by Liu and associates (2005a). The onion epidermal cells then were stained with propidium iodide $(10 \mu \mathrm{g} / \mathrm{ml})$ for nuclei and were observed with a Leica TCS SP2 AOBS confocal microscope (Leica Microsystems, Heidelberg GmbH, Germany).

\section{Yeast one-hybrid assays.}

The interaction of OsWRKY13 protein with W or W-like box in a promoter region (Supplemental Table 3) was examined using a yeast one-hybrid assay according to the manufacturer's protocol (Clontech Yeast Protocols Handbook, BD Biosciences Clontech, Mountain View, CA, U.S.A.). The DNA fragment (W17) without a W or W-like box from the promoter region of OsWRKY13 was used as control. Positive interactions were verified by growing on SD-Leu-Trp-His agar medium.

\section{Gel mobility shift assay.}

Rice leaves were harvested from six-leaf stage rice for isolation of nuclear extract, essentially using the method reported by Marzabal and associates (1998). For recombinant proteins, the full-length cDNA of OsWRKY13 was cloned into pDEST15 to generate GST-OsWRKY13 through a GATEWAY system (Invitrogen life technologies, Carlsbad, CA, U.S.A.). The fusion construct was then introduced into BL21-CodonPlus(DE3)-RP Escherichia coli (Stratagene, La Jolla, CA, U.S.A.). The GST-OsWRKY13 fusion protein was purified using MagneGST glutathione particles (Promega, Madison, WI, U.S.A.) according to the manufacturer's protocol. Gel mobility shift assay was applied as described previously (Urao et al. 1993). The synthetic DNA probe harboring a W-like box from the promoter of $O s W R K Y 13$ was generated by mixing the two complementary oligonucleotides (TTTTATTTGACGTTC TAGA and TCTAGAACGTCAAATAAAA).

\section{Quantification of SA.}

Each sample was harvested from four to eight plants at the booting stage. The SA samples were extracted with slight modification of the procedure of Bowling and associates (1994). In brief, leaves were ground under liquid nitrogen. Three replicates of each ground sample weighing 300 to 1,000 $\mathrm{mg}$ (exact weights were recorded) were placed in $3 \mathrm{ml}$ of $80 \%$ methanol supplemented with $1 \mu \mathrm{g}$ of naphthaleneacetic acid standard as internal control and were left to extract at $4{ }^{\circ} \mathrm{C}$ for $24 \mathrm{~h}$ in the dark. The average recovery rate of the procedure was approximately $75 \%$. The organic extracts containing the free SA were analyzed by high-performance liquid chromatography (Agilent 1100; Agilent Technologies, Palo Alto, CA, U.S.A.) with an Agilent C18 Zorbax ODS column $(250 \times 5$ $\mathrm{mm})$ run at $25^{\circ} \mathrm{C}$ in $50 \%$ water $\left(0.1 \% \mathrm{H}_{3} \mathrm{PO}_{4}\right)$ and $50 \%$ methanol with a flow rate of $0.6 \mathrm{ml} / \mathrm{min}$. SA was detected using a 230-nm wavelength. The aqueous phase containing the conjugated SA was acidified with $\mathrm{HCl}$ to $\mathrm{pH} 1$ and was boiled for 30 min to release SA from any acid-labile conjugated forms. The released free SA was then extracted with the organic mixture and was analyzed.

\section{Chemical treatments.}

Chemical treatments were applied according to the methods of Agrawal and associates (2000). Minghui 63 was grown in a greenhouse at $25^{\circ} \mathrm{C}, 70 \%$ relative humidity, and a 12 -h photoperiod for 21 days. Approximately 2-cm long leaf segments were cut from the fully expanded leaves and were floated on $30 \mathrm{ml}$ of solution containing the test compound in covered sterile petri dishes. Leaf segments wounded by cutting and floated on deionized water served as control. The experiments were repeated twice. SA, JA, ethephon, BTH, and INA were purchased from Sigma (St. Louis). The concentration of working solutions was $100 \mu \mathrm{M}$.

\section{Sequence analysis.}

A $1.5-\mathrm{kb}$ sequence upstream of the coding region of each defense-related gene was analyzed to identify the cis-regulatory elements $\mathrm{W}$ or $\mathrm{W}$-like box using the computer program PLACE Signal Scan (a database of plant cis-acting regulatory DNA elements). The $\mathrm{W}$ and $\mathrm{W}$-like boxes were classified according to the published criteria (Eulgem et al. 2000; Maleck et al. 2000).

\section{ACKNOWLEDGMENTS}

We thank the Center for the Application of Molecular Biology to International Agriculture (Canberra, Australia) for providing vectors pCAMBIA1300 and pCAMBIA1301 and Q. Shen of the University of Nevada (Reno, U.S.A.) for providing the pDT01208005 vector. This work was supported by grants from the National Program on the Development of Basic Research in China and the National Natural Science Foundation of China.

\section{LITERATURE CITED}

Agrawal, G. K., Jwa, N. S., and Rakwal, R. 2000. A novel rice (Oryza sativa $\mathrm{L}$.) acidic PR1 gene highly responsive to cut, phytohormones, and protein phosphatase inhibitors. Biochem. Biophys. Res. Commun. 274:157-165

Agrawal, G. K., Rakwal, R., and Jwa, N. S. 2001. Differential induction of three pathogenesis-related genes, PR10, PR1b and PR5 by the ethylene generator ethephon under light and dark in rice (Oryza sativa L.) seedlings. J. Plant Physiol. 158:133-137.

Asai, T., Tena, G., Plotnikova, J., Willmann, M. R., Chiu, W. L., GomezGomez, L., Boller, T., Ausubel, F. M., and Sheen, J. 2002. MAP kinase signaling cascade in Arabidopsis innate immunity. Nature 415:977-983.

Bowling, S. A., Guo, A., Cao, H., Gordon, A. S., Klessig, D. F., and Dong, X. 1994. A mutation in Arabidopsis that leads to constitutive expression of systemic acquired resistance. Plant Cell 6:1845-1857.

Chen, C., and Chen, Z. 2002. Potentiation of developmentally regulated plant defense response by AtWRKY18, a pathogen-induced Arabidopsis transcription factor. Plant Physiol. 129:706-716.

Chen, H., Chen, B., Zhang, D., Xie, Y., and Zhang, Q. 2001. Pathotypes of Pyricularia grisea in rice fields of central and southern China. Plant Dis. 85:843-850.

Chern, M. S., Fitzgerald, H. A., Yadav, R. C., Canlas, P. E., Dong, X., and Ronald, P. C. 2001. Evidence for a disease-resistance pathway in rice similar to the NPR1-mediated signaling pathway in Arabidopsis. Plant J. 27:101-113.

Chern, M., Fitzgerald, H. A., Canlas, P. E., Navarre, D. A., and Ronald, P. C. 2005. Overexpression of a rice NPR1 homolog leads to constitutive activation of defense response and hypersensitivity to light. Mol. PlantMicrobe Interact. 18:511-520.

Deslandes, L., Olivier, J., Theulieres, F., Hirsch, J., Feng, D. X., BittnerEddy, P., Beynon, J., and Marco, Y. 2002. Resistance to Ralstonia solanacearum in Arabidopsis thaliana is conferred by the recessive $R R S 1-R$ gene, a member of a novel family of resistance genes. Proc. Natl. Acad. Sci. U.S.A. 99:2404-2409.

Durrant, W. E., and Dong, X. 2004. Systemic acquired resistance. Annu. Rev. Phytopathol. 42:185-209.

Eulgem, T., Rushton, P.J., Robatzek, S., and Somssich, I. E. 2000. The 
WRKY superfamily of plant transcription factors. Trends Plant Sci. 5:199-206.

Falk, A., Feys, B. J., Frost, L. N., Jones, J. D. G., Daniels, M. J., and Parker, J. E. 1999. EDS1, an essential component of $R$ gene mediated disease resistance in Arabidopsis has homology to eukaryotic lipases. Proc. Natl. Acad. Sci. U.S.A. 96:3292-3297.

Felton, G. W., and Korth, K. L. 2000. Trade-offs between pathogen and herbivore resistance. Curr. Opin. Plant Biol. 3:309-314.

Feys, B. J., Moisan, L. J., Newman, M. A., and Parker, J. E. 2001. Direct interaction between the Arabidopsis disease resistance signaling proteins, EDS1 and PAD4. EMBO (Eur. Mol. Biol. Organ.) J. 20:54005411.

Glazebrook, J. 2001. Genes controlling expression of defense responses in Arabidopsis: 2001 status. Curr. Opin. Plant Biol. 4:301-308.

Gozzo, F. 2003. Systemic acquired resistance in crop protection: From nature to a chemical approach. J. Agric. Food Chem. 51:4487-4503.

Hammond-Kosack, K. E., and Parker, J. E. 2003. Deciphering plant-pathogen communication: Fresh perspectives for molecular resistance breeding. Curr. Opin. Biotechnol. 14:177-193.

Hu, W., and Ma, H. 2006. Characterization of a novel putative zinc finger gene MIF1: Involvement in multiple hormonal regulation of Arabidopsis development. Plant J. 45:399-422.

Jirage, D., Tootle, T. L., Reuber, T. L., Frost, L. N., Feys, B. J., Parker, J. E., Ausubel, F. M., and Glazebrook, J. 1999. Arabidopsis thaliana $P A D 4$ encodes a lipase-like gene that is important for salicylic acid signaling. Proc. Natl. Acad. Sci. U.S.A. 96:13583-13588.

Journot-Catalino, N., Somssich, I. E., Roby, D., and Kroj, T. 2006. The transcription factors WRKY11 and WRKY17 act as negative regulators of basal resistance in Arabidopsis thaliana. Plant Cell (in press).

Kauffman, H. E., Reddy, A. P. K., Hsieh, S. P. Y., and Merca, S. D. 1973. An improved technique for evaluating resistance of rice varieties to Xanthomonas oryzae. Plant Dis. Rep. 57:537-541.

Kim, K.-C., Fan, B., and Chen, Z. 2006. Pathogen-induced Arabidopsis WRKY7 is a transcriptional repressor and enhances plant susceptibility to Pseudomonas syringae. Plant Physiol. 142:1180-1192.

Kinkema, M., Fan, W., and Dong, X. 2000. Nuclear localization of NPR1 is required for activation of $\mathrm{PR}$ gene expression. Plant Cell 12:23392350.

Lee, H. I., Leon, J., and Raskin, I. 1995. Biosynthesis and metabolism of salicylic acid. Proc. Natl. Acad. Sci. U.S.A. 92:4076-4079.

Li, J., Brader, G., and Palva, E. T. 2004. The WRKY70 transcription factor: A node of convergence for jasmonate-mediated and salicylatemediated signals in plant defense. Plant Cell 16:319-331.

Lin, Y. J., and Zhang, Q. 2005. Optimising the tissue culture conditions for high efficiency transformation of indica rice. Plant Cell Rep. 23:540547.

Liu, G., Holub, E. B., Alonso, J. M., Ecker, J. R., and Fobert, P. R. 2005a. An Arabidopsis NPR1-like gene, NPR4, is required for disease resistance. Plant J. 41:304-318.

Liu, X. Q., Bai, X. Q., Qian, Q., Wang, X. J., Chen, M. S., and Chu, C. C. 2005b. OsWRKY03, a rice transcriptional activator that functions in defense signaling pathway upstream of OsNPR1. Cell Res. 15:593-603.

Maleck, K., Levine, A., Eulgem, T., Morgan, A., Schmid, J., Lawton, K. A., Dangl, J. L., and Dietrich, R. A. 2000. The transcriptome of Arabidopsis thaliana during systemic acquired resistance. Nat. Genet. 26:403-410.

Marzabal P., Busk P. K., Ludevid M. D., and Torrent M. 1998. The bifactorial endosperm box of $\gamma$-zein gene: Characterisation and function of the $\mathrm{Pb} 3$ and GZM cis-acting elements. Plant J. 16:41-52.

Mauch, F., Mauch-Mani, B., Gaille, C., Kull, B., Haas, D., and Reimmann, C. 2001. Manipulation of salicylate content in Arabidopsis thaliana by the expression of an engineered bacterial salicylate synthase. Plant J. 25:67-77.

Mei, C., Qi, M., Sheng, G., and Yang, Y. 2006. Inducible overexpression of a rice allene oxide synthase gene increases the endogenous jasmonic acid level, $P R$ gene expression, and host resistance to fungal infection. Mol Plant-Microbe Interact 19:1127-1137.

Noutoshi, Y., Ito, T., Seki, M., Nakashita, H., Yoshida, S., Marco, Y., Shirasu, K., and Shinozaki, K. 2005. A single amino acid insertion in the WRKY domain of the Arabidopsis TIR-NBS-LRR-WRKY-type dis- ease resistance protein SLH1 (sensitive to low humidity 1) causes activation of defense responses and hypersensitive cell death. Plant $\mathbf{J}$. 43:873-888.

Pieterse, C. M., and Van Loon, L. C. 2004. NPR1: The spider in the web of induced resistance signaling pathways. Curr. Opin. Plant Biol. 7:456464.

Qu, L. J., and Zhu, Y. X. 2006. Transcription factor families in Arabidopsis: Major progress and outstanding issues for future research. Curr. Opin. Plant Biol. 9:544-549

Robatzek, S., and Somssich, I. E. 2002. Targets of AtWRKY6 regulation during plant senescence and pathogen defense. Genes Dev. 16:11391149.

Spoel, S. H., Koornneef, A., Claessens, S. M., Korzelius, J. P., Van Pelt, J A., Mueller, M. J., Buchala, A. J., Metraux, J. P., Brown, R., Kazan, K., Van Loon, L. C., Dong, X., and Pieterse, C. M. 2003. NPR1 modulates cross-talk between salicylate- and jasmonate-dependent defense pathways through a novel function in the cytosol. Plant Cell 15:760-770.

Thomma, B., Eggermont, K., Penninckx, I., Mauch-Mani, B., Vogelsang, R., Cammue, B. P. A., and Broekaert, W. F. 1998. Separate jasmonatedependent and salicylate-dependent defense-response pathways in Arabidopsis are essential for resistance to distinct microbial pathogens. Proc. Natl. Acad. Sci. U.S.A. 95:15107-15111.

Turck, F., Zhou, A., and Somssich, I. E. 2004. Stimulus-dependent, promoter-specific binding of transcription factor WRKY1 to its native promoter and the defense-related gene $P c P R-1$ in parsley. Plant Cell $16: 2573-2585$.

Tusher, V. G., Tibshirani, R., and Chu, G. 2001. Significance analysis of microarrays applied to the ionizing radiation response. Proc. Natl. Acad. Sci. U.S.A. 98:5116-5121.

Urao T., Yamaguchi-Shinozaki K., Urao S., and Shinozaki K. 1993. An Arabidopsis myb homolog is induced by dehydration stress and its gene product binds to the conserved MYB recognition sequence. Plant Cell 5:1529-1539.

Wen, N., Chu, Z., and Wang, S. 2003. Three types of defense-responsive genes are involved in resistance to bacterial blight and fungal blast diseases in rice. Mol. Genet. Genomics 269:331-339.

Wildermuth, M. C., Dewdney, J., Wu, G., and Ausubel, F. M. 2001. Isochorismate synthase is required to synthesize salicylic acid for plant defence. Nature 414:562-565.

Xie, Z., Zhang, Z. L., Zou, X., Huang, J., Ruas, P., Thompson, D., and Shen, Q. J. 2005. Annotations and functional analyses of the rice WRKY gene superfamily reveal positive and negative regulators of abscisic acid signaling in aleurone cells. Plant Physiol. 137:176-189.

$\mathrm{Xu}, \mathrm{X}$., Chen, C., Fan, B., and Chen, Z. 2006. Physical and functional interactions between pathogen-induced Arabidopsis WRKY18, WRKY40 and WRKY60 transcription factors. Plant Cell 18:1310-1326.

Yang, P., Chen, C., Wang, Z., Fan, B., and Chen, Z. 1999. A pathogen- and salicylic acid-induced WRKY DNA-binding activity recognizes the elicitor response element of the tobacco class I chitinase gene promoter. Plant J. 18:141-149.

Zhang, Y., Fan, W., Kinkema, M., Li, X., and Dong, X. 1999. Interaction of NPR1 with basic leucine zipper protein transcription factors that bind sequences required for salicylic acid induction of the PR-1 gene. Proc. Natl. Acad. Sci. U.S.A. 96:6523-6528.

Zheng, Z., Qamar, S. A., Chen, Z., and Mengiste, T. 2006. Arabidopsis WRKY33 transcription factor is required for resistance to necrotrophic fungal pathogens. Plant J. 48:592-605.

Zhao, J., Davis, L. C., and Verpoorte, R. 2005. Elicitor signal transduction leading to production of plant secondary metabolites. Biotechnol. Adv. 23:283-333.

Zhou, B., Peng, K., Chu, Z., Wang, S., and Zhang, Q. 2002. The defenseresponsive genes showing enhanced and repressed expression after pathogen infection in rice (Oryza sativa L.). Science in China (Ser. C) 45:450-467.

\section{AUTHOR-RECOMMENDED INTERNET RESOURCE}

PLACE Signal Scan database:

www.dna.affrc.go.jp/PLACE/signalscan.html 